\title{
Towards faster techno-economic evaluation of network scenarios via a modular network equipment database
}

\author{
Bram Naudts \\ Department of \\ Information Technology \\ Ghent University \\ Ghent, Belgium \\ Email: bram.naudts@intec.ugent.be
}

\author{
Sofie Verbrugge \\ Department of \\ Information Technology \\ Ghent University \\ Ghent, Belgium \\ Email: sofie.verbrugge@intec.ugent.be
}

\author{
Didier Colle \\ Department of \\ Information Technology \\ Ghent University \\ Ghent, Belgium \\ Email: didier.colle@intec.ugent.be
}

\begin{abstract}
Techno-economic analysis is used to evaluate technological solutions in different business environments via a simulation based approach. Four steps can be observed in techno-economic analysis: scope definition, modeling of costs and revenues, evaluation of the different solutions and refinement of the analysis. During the scoping step, the boundaries of the analysis are determined, the problem is divided into sub problems and a list of input values is collected. The collection of complete and precise inputs is of the greatest importance as faulty data leads to unreliable results (garbage in garbage out). Today, due to the lack of a structured way of sharing input values, inputs need to be collected on a case-by-case basis. Although a valid approach, it remains a time intensive process prone to errors.
\end{abstract}

This paper therefore proposes a modular network equipment database containing a set of generic equipment such as an IP/MPLS router, a DWDM node, a fiber link and a twisted pair cable. Each of these is represented as a modular block model and described by a set of attributes and a set of drivers which are used to chain one network equipment's block model to one or more others. A complex network can as such be modeled as a chain of equipment block models which are determined by a set of drivers. As a consequence, the collection of inputs can be fast tracked, the problem of missing inputs can be partially resolved and cross-case comparison becomes possible. To make things more concrete, we illustrate this approach via the evaluation of a network scenario in which a network service provider expands its network with an extra core location.

\section{INTRODUCTION}

The results in a techno-economic analysis depend heavily on the quality of the input values gathered. Bad data will lead to unreliable results and misinterpretation. When one wants to investigate a network scenario, for example the cost of expanding a transport network, the result will depend on the equipment configuration, the obtained price points, the constraining factors and the compatibility between equipment. Unfortunately each of these can be quite challenging. Due to cryptic descriptions of how to build a device out of components, equipment configuration may turn into a headscratching journey through vendor web pages, analyst reports and technical documentation. Once the first challenge is resolved, the second occurs: finding reliable cost values for the components. This can be a hassle due to the unavailability of data (e.g. confidential data) or the ever-changing formats used to provide data (e.g. changing IDs, components being pulled, different versions of the same component, etc.). As a result of incomplete information, one may need to restart the process entirely (e.g. with another vendor) or rely on estimates (with the risk of bad data leading to unreliable results). But let's consider we managed (after a couple of iterations) to get a configuration with reliable (cost) data points. The next challenge has to be faced: the determination of the capacity of a network node or link (e.g. number of subscribers a server can support, storage and capacity, etc.). Hopefully, test data can be found via analyst reports or one may need to rely on the specifications as listed on the data sheets (which are often under ideal conditions). Finally, one must cope with the difficulties that may appear when connecting equipment from different vendors (or even of the same vendor) to create a network of interconnected nodes and links as not all equipment may be compatible. Unfortunately, their seems to be no easy way to overcome this time-intensive procedure of gathering input data. Of course, lack of data can be resolved by setting up a central location where cost data can be shared. But even with the price lists of vendors available one still needs to invest time to find out how to construct a device, to understand compatibility issues and to understand possible constraints.

In this paper, we propose a way to tackle this challenge by proposing a structured way of representing data which can be used to fast-track techno-economic analysis. By revisiting the challenges describe above, we extracted a set of requirements:

Req. 1 at the lowest level of abstraction, it should visualize modular network equipment that is composed of individual components in an easy to understand format

Req. 2 it should be able to specify the constraints that determine the dimensioning of modular network equipment in a network scenario

Req. 3 the model should be able to contain cost aspects that are driven by the deployment, operation and tear down of modular network equipment

Req. 4 it should be possible to vary the input parameters that drive the dimensioning process of a network scenario

Req. 5 it should allow the chaining of modular network 
equipment for the evaluation of complex network scenarios

Req. 6 it should allow to consistently aggregate modular network equipment at different levels of detail in order to analyze them from diverse points of view

Req. 7 it should be extensible and exchangeable

When each of these requirements are met, the resulting models will be exchangeable. As such, the models will be available as a reference input for different studies to increase their repeatability. In addition it will be easier to compare results between vendors and between studies.

The remainder of this paper starts with proposing a general approach of tackling the requirements listed above (Section II). This approach uses Equipment Coupling Modeling Notation (ECMN) models and comprises a visual notation and a set of attributes linked to the building blocks. In Section III we provide examples of ECMN models for an IP/MPLS router, an OXC and an optical link. In Section IV we show how these ECMN models can be chained together via a case study of two network scenarios. In the last section, we summarize our work and propose future work (Section V).

\section{GENERAL APPROACH: A MODULAR NETWORK EQUIPMENT DATABASE}

The main purpose of this work is to fast track technoeconomic evolution of network scenarios and to increase comparability of its results. To do so, we propose a twofold approach which consists of a modeling notation to describe network equipment and an (extensible) set of data attributes. Both are described below:

Req. 1 Equipment Coupling Modeling Notation (ECMN) is used to visualize the components of modular network equipment in an easy to understand, human readable format. This modeling notation was originally developed to calculate the cost of installation of the equipment in a central office [1], [2]. It exploits the hierarchical structure of a central office to determine the amount of equipment that needs to be installed (dimensioning) and visualizes via a graph structure how equipment is linked together as is illustrated in Figure 1 for a central office. Clearly the same modeling notation can be used for dimensioning routers, servers, fiber links or any other equipment.

Req. 2 In ECMN, a tree structure is graphically drawn indicating the equipment that needs to be installed. To specify the constraints that determine the dimensioning, granularity factors are used on the connections that link the equipment (i.e. one shelf can accommodate at max three OLT cards).

Req. 3 Every block in an ECMN model can contain a set of attributes. These (optional) attributes include the initial equipment purchase cost, installation cost, operational cost (e.g. power consumption and space), maintenance cost, repair cost and tear down cost. The input values for these attributes are represented as a time function which is a list of values. Each value represents the input parameter for a certain time period.

Req. 4 In ECMN, parameters referred to as drivers should also be linked to the entire model (e.g. customers in Figure 1). These drivers initiate the calculation and are connected to the lowest equipment types in the graphical tree. Because of the hierarchical structure of the model, the calculation can propagate from the lowest branches up to the higher branches, thereby allowing calculating the entire tree bottom-up.

Req. 5 Once an ECMN model is calculated based on the provided input drivers, the output(s) of the calculation can be used as a driver for linked ECMN models. For example, the number of Small Form-factor Pluggable (SFP) transceivers of a router could be the driver for an ECMN model that represents a fiber connection. Similarly, the number of fibers could be the driver for an ECMN model that represents an optical crossconnect (OXC), etc.

Req. 6 Depending on the goal, the level of detail should be adjustable. ECMN therefore defines sub models to provide abstraction of details. Because an equipment model is inherently hierarchical, it can be stacked in a hierarchical manner. As such, a sub model is used for more intuitive visualization and modeling. At the lowest level of detail, vendor price points can be added to a model by adding a vendor-specific field to the list of attributes.

Req. 7 A model can be exported in a data-interchange format such as JSON or XML and is stored in a database for future reference or usage. As such it is easy to reuse existing models and to add newly developed ones. It is also possible to update existing models to include new attributes.

A database of ECMN models resolves challenges such as the lack of structured data, lack of comparability of results (e.g. between studies and between vendors) and the lack of repeatability. A major drawback of this approach is that the initial development of an ECMN model can be time-intensive. The population of the database of ECMN models is therefore a major challenge. However, once a critical amount of models is reached it will be able to attract more users who add their own ECMN models and increase the total value. As a start for the database, we present four ECMN models in the next section.

\section{EXEMPLARY ECMN MODELS}

The ECMN models are organized corresponding to layers 1 (L1) to layers 3 (L3) of the open systems interconnection (OSI) reference model:

- L3/L2 Internet Protocol (IP)/Multiprotocol Label Switching (MPLS) router

- L1 Optical cross connect

- $\quad$ L1 Fiber line

For the different components in each layer, we limit the list of attributes that are part of the ECMN model to pricing values of the purchase price of (a complete ECMN model would also include values for power consumption, mean time between failure, etc.). One exception is the price of dark fiber as this is not the purchase price but the yearly lease for $1 \mathrm{~km}$ of dark fiber. Note that we work with price (before discounts) and not cost values. Nevertheless, in general the terms cost and price are used interchangeably. The price points that are obtained from [3] are based on the assumption that 1 STRONGEST Cost Unit (SCU) corresponds with $\$ 4,000$. 


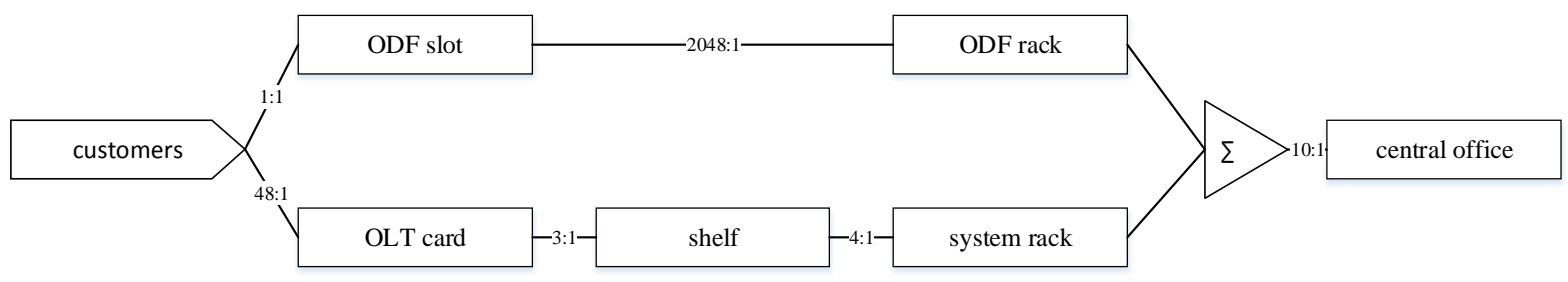

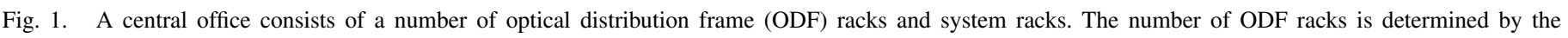

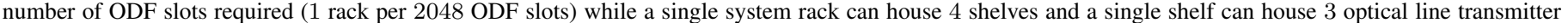

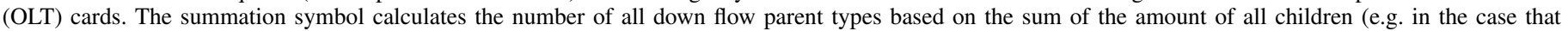
$5 \mathrm{ODF}$ racks and 5 system racks are in use, one extra rack of either type would require an extra central office.)

\section{A. L3/2 IP/MPLS Router}

An IP/MPLS router performs IP routing and MPLS forwarding in the electrical domain. Two different flavors of MPLS exist to establish connection-oriented communication across a connectionless packet network: first, MPLS with traffic engineering extensions (MPLS-TE) with resource reservation protocol - traffic engineering extensions (RSVP-TE) signaling to construct end-to-end label switched paths (LSPs) and second, MPLS using label distribution protocol (LDP) signaling to construct the LSPs. Depending on how it is configured, a router port can forward packets on IP addresses, on MPLS labels or on Ethernet media access control (MAC) addresses. This type of routers are typically deployed in aggregation- and core networks.

The ECMN model of this router is based on the electrical layer node building blocks as proposed by the authors of [3] an illustrated in Figure 2. These building blocks include the basic node which consists of the the physical and mechanical assembly, power supplies and cooling, the switch matrix, and control- and data plane software. A basic node provides a specified number of bidirectional slots with a maximum transmission speed. Into each slot, a line card can be installed with the corresponding or a lower speed (e.g. a 100Gbps slot can house a 10/40/100Gbps line card). Each line card has a number of specified ports with a specified speed. In each port, a transceiver can be plugged in which combines a transmitter and a receiver.

In a first step, the building blocks of Figure 2 are mapped to the equipment coupling modeling notation and a tree structure is graphically drawn indicating the components that need to be installed in the IP/MPLS router, while linking them using granularity factors. In a second step, the needed input parameters are defined per component (e.g. cost points) and on the entire model (the drivers, e.g. the number of $\mathrm{xGbE}$ connected cables, $\mathrm{x} \in\{1,10,40,100\})$. These drivers are linked to the lowest equipment types in the graphical tree (the number of connected cables drives the amount of transceivers), they serve as such as drivers for the higher branches, thereby allowing calculations of the entire tree bottom-up. The models allows calculating both amounts of each equipment type, as well as their costs. The outcome is illustrated in Figure 3a. The basic node block contains a sub model which is illustrated in Figure 4. Note that the granularity factors of Figure $3 \mathrm{a}$ are to be completed for a vendor specific implementation. Figures $3 \mathrm{~b}, 3 \mathrm{c}$ and $3 \mathrm{~d}$ illustrate this for the Cisco ASR 9001, Cisco

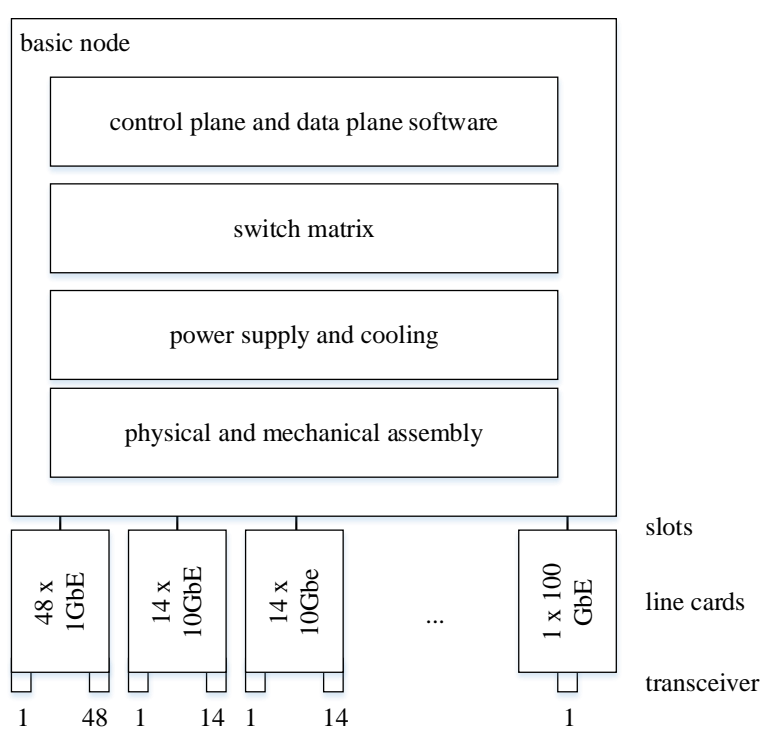

Fig. 2. Schematic representation of the main building blocks of an electrical node such as an IP/MPLS router: basic node, line cards and transceivers.

ASR 9006 and Cisco ASR 9010 router respectively [4], [5], [6]. Cisco offers a different number of ports for the same type of line card (e.g. a 10 gigabit Ethernet $(\mathrm{GbE})$ linecard with 2 ports and one with 4 ports), this information is added via the granularity factors. To complete the ECMN model, an overview of the price points for each component is given in Table I.

\section{B. L1 Optical Cross Connect}

The second ECMN model describes an optical cross connect (OXC) which is used in carrier networks to switch optical signals in a fiber optic network. In the optical layer (dense) wavelength division multiplexing ((D)WDM) is used to transmit data over long-haul distances using multiple wavelength channels within optical fibers. We limit the discussion to the traditional system in which each channel fits into the International Telecommunication Union - Telecommunication Standardization Sector (ITU-T) $50 \mathrm{Ghz}$ grid and focus on the description of an OXC. 
a.

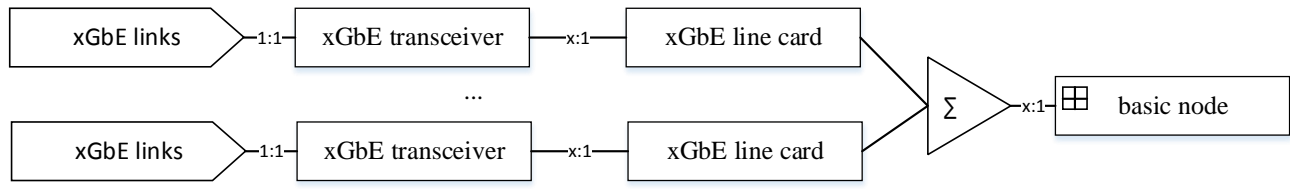

b.

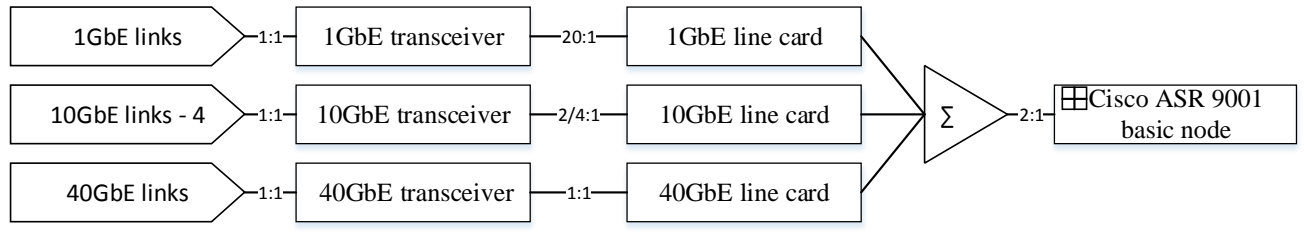

c.

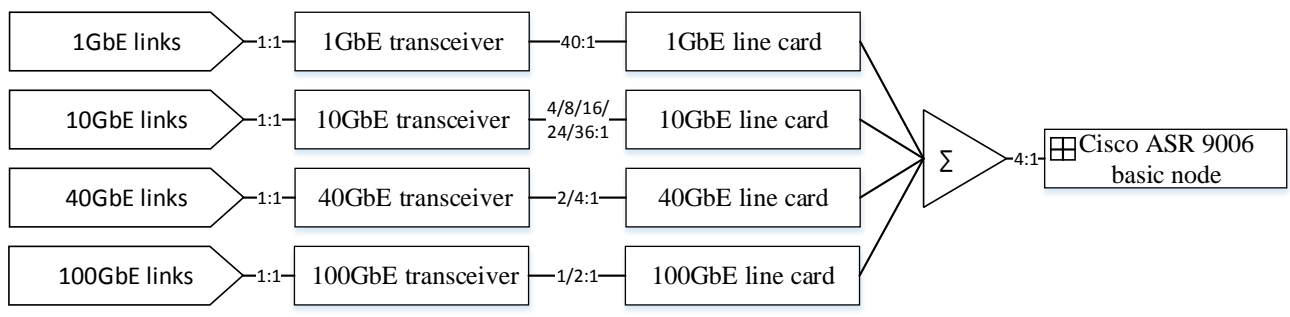

d.

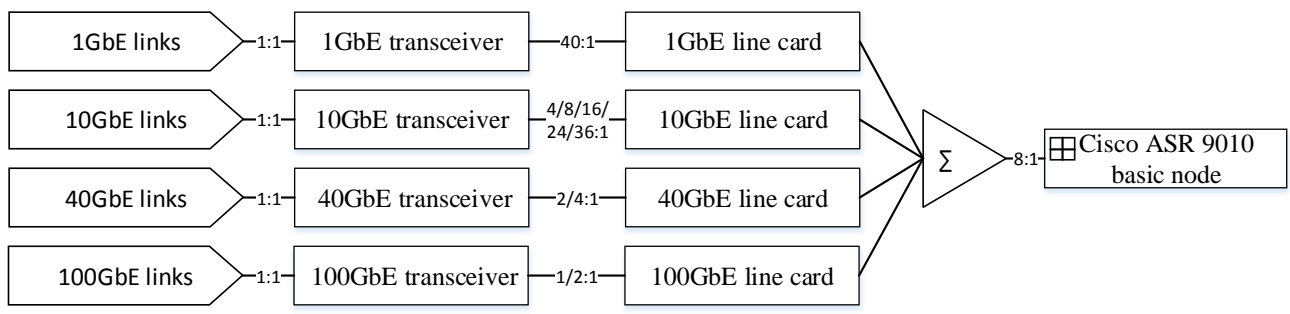

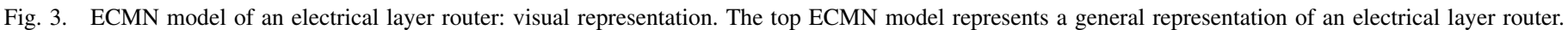

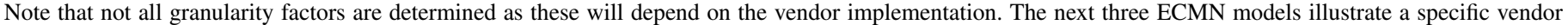
implementation: Cisco ASR 9001, CISCO ASR 9006 and CISCO ASR 9010.

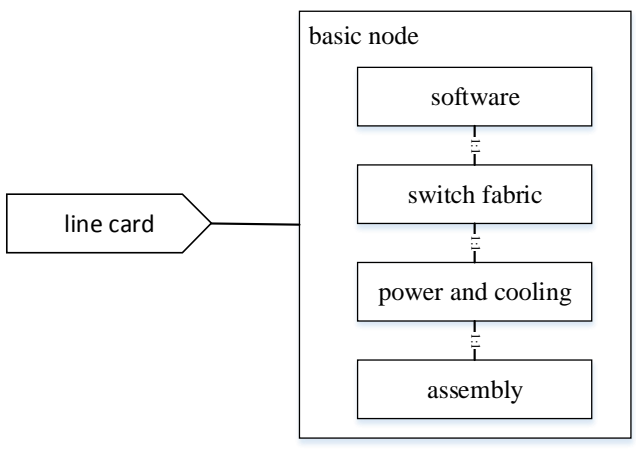

Fig. 4. ECMN submodel of the basic node: visual representation. By defining submodels it is possible to abstract detail when useful.
TABLE I. BUILDING BLOCKS FOR AN IP/MPLS ECMN MODEL.

\begin{tabular}{lrr}
\hline component & \multicolumn{1}{l}{ cost } & source \\
\hline basic node 2 slots (similar to ASR 9001) & $\$ 110,000.00$ & {$[7]$} \\
basic node 4 slots (similar to ASR 9006) & $\$ 120,000.00$ & {$[7]$} \\
basic node 8 slots (similar to ASR 9010) & $\$ 140,000.00$ & {$[7]$} \\
$20 \times 1$ GbE line card & $\$ 15,000.00$ & {$[7]$} \\
$40 \times 1$ GbE line card & $\$ 65,000.00$ & {$[7]$} \\
$2 \times 10 \mathrm{GbE}$ line card & $\$ 15,000.00$ & {$[7]$} \\
$4 \times 10 \mathrm{GbE}$ line card & $\$ 35,000.00$ & {$[7]$} \\
$8 \times 10 \mathrm{GbE}$ line card & $\$ 65,000.00$ & {$[7]$} \\
$16 \times 10 \mathrm{GbE}$ line card & $\$ 170,000.00$ & {$[7]$} \\
$24 \times 10 \mathrm{GbE}$ line card & $\$ 400,000.00$ & {$[7]$} \\
$36 \times 10 \mathrm{GbE}$ line card & $\$ 480,000.00$ & {$[7]$} \\
$1 \times 40 \mathrm{GbE}$ line card & $\$ 40,000.00$ & {$[7]$} \\
$2 \times 40 \mathrm{GbE}$ line card & $\$ 75,000.00$ & {$[7]$} \\
$4 \times 40 \mathrm{GbE}$ line card & $\$ 150,000.00$ & {$[7]$} \\
$1 \times 100 \mathrm{GbE}$ line card & $\$ 200,000.00$ & {$[7]$} \\
$2 \times 100 \mathrm{GbE}$ line card & $\$ 400,000.00$ & {$[7]$} \\
1 GbE transceiver & $\$ 2,000.00$ & {$[7]$} \\
$10 \mathrm{GbE}$ transceiver & $\$ 10,000.00$ & {$[7]$} \\
$40 \mathrm{GbE}$ transceiver & $\$ 22,400.00$ & {$[7]$} \\
$100 \mathrm{GbE}$ transceiver & $\$ 56,000.00$ & {$[7]$} \\
\hline
\end{tabular}

An OXC is a WDM node which is remotely configurable (therefore also commonly denoted as a reconfigurable optical 
add/drop multiplexer (ROADM)). A (D)WDM OXC is built differently than an IP/MPLS router. As a first step in the building of an OXC, one has to choose the number of ports that are connected to other nodes via fiber (i.e. the nodal degree). Second, a choice has to be made with respect to the general optical switching capabilities [8]:

- $\quad$ basic ROADM implementation: channels are routed to a particular degree (each degree represents a direction in which the node connects to another node) or to the wavelength add/drop structure. Reconfigurability is limited to routing the wavelength, and the add/drop structure is fixed (referred to as a colored design).

- colorless: any wavelength can be assigned to an add/drop port, in this design each degree of the node has its own unique add/drop structure. As such, moving a wavelength to another degree requires physically moving the service to different ports on the desired degree.

- colorless and directionless: any channel can be directed to any degree on the ROADM (any client port can be connected to any node/line port), the same channel color can be used only once per add/drop structure.

- colorless, directionless and contentionless: the node architecture does not imply any wavelength switching contention inherent the node, a transmitter can be assigned to any wavelength as long as the number of channels with the same wavelength is not more than the nodal degree.

Third, the cost for the OXC depends on how many wavelength channels can be dropped or added (note that the number of wavelength channels has to be the same for the whole modeled WDM layer). Finally, to actually transmit client traffic, transponders have to be installed at these nodes. The number of transponders corresponds with the number of wavelength channels. A huge number of configurations are possible when combining the elements of the model. As an example, we develop an ECMN model for a colorless, directionless and contentionless ROADM with a nodal degree of 4 with 80,88 or 96 channels. The OXC model is based on [3] and is illustrated in Figure 5. The OXC consists of wavelength selective switches (WSS). A 1 x $N$ WSS is a device that is able to switch a selected wavelength or wavelengths from an input port to an output port. The number of WSS on the express (Figure 5a) path as well as the amplifiers are driven by the nodal degree. On the add/drop path (Figure 5b), a two-stage add-drop chain is used, composed of a couple of $9 \times 9$ WSSs at the first stage and two pairs of 1x20 WSS modules at the second stage (the add-drop capacity can grow up to 180 bidirectional lambdas when a maximum of 7 additional WSS module-couples are added). The number of 9x9-WSSs is driven by the maximum of two factors:

- $\quad$ the nodal degree (at least 2 per nodal degree),

- the number of 1x20 WSSs (2 9x9-WSSs per 9 1x20WSSs).

The number of $1 \times 20-W S S s$ is driven by the maximum of two factors:
- the product of the nodal degree with the add/drop capacity in percentage with granularity 20 $(\mathrm{AD}(\%) / 20 \%)$,

- the sum of the transponders and colored electrical layer ports on the other.

Finally, the number of transponders depends on the number of connections with electrical equipment (e.g. from an IP/MPLS node) and the installed transceivers (transponders are not required for connections with electrical switches equipped with colored transceivers, gray transceivers require a transponder). The ECMN model is illustrated in Figure 6 and cost points are given in Table II.

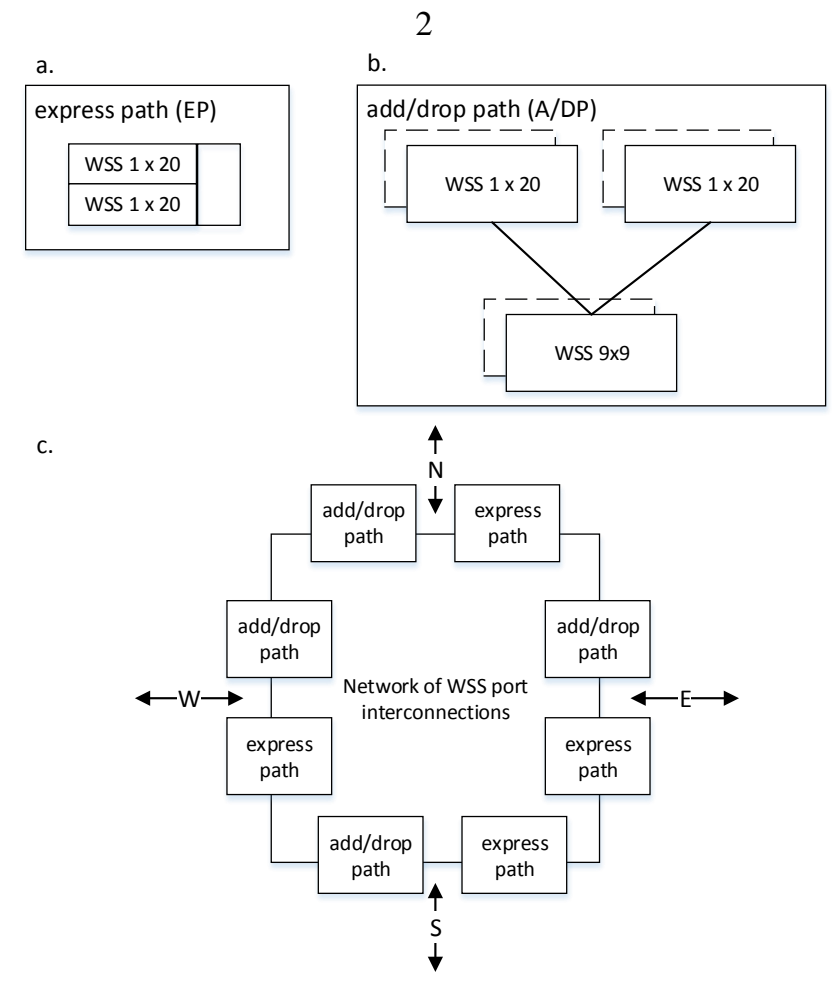

Fig. 5. Schematic representation of a colorless, directionless and contentionless optical cross connect. Figure 5a shows the express path which consists of a pair of WSSs. Figure $5 \mathrm{~b}$ shows the add/drop path which consists of a two-stage design of WSSs. Figure 5c shows the final design of an OXC with a nodal degree of 4 .

TABLE II. BUILDING BLOCKS FOR AN OXC ECMN MODEL.

\begin{tabular}{lrr}
\hline component & cost & source \\
\hline wavelength selective switch $(1 \times 20)$ & $24,000.00$ & {$[3]$} \\
wavelength selective switch $(9 \times 9)$ & $192,000.00$ & {$[3]$} \\
amp pre & $3,200.00$ & {$[3]$} \\
amp boost & $3,200.00$ & {$[3]$} \\
10G transponder $(750 \mathrm{~km} / 2000 \mathrm{~km})$ & $3,600.00 / 4,000.00$ & {$[3]$} \\
40G transponder & $24,000.00$ & {$[3]$} \\
100G transponder & $60,000.00$ & {$[3]$} \\
400G transponder & $68,000.00$ & {$[3]$} \\
\hline
\end{tabular}

\section{L1 Optical Fiber}

A (D)WDM OXC transmits data over long-haul distances to another OXC via L1 optical fibers. The optical signal exits the $\mathrm{OXC}$ at a defined port and reaches its destination which can 


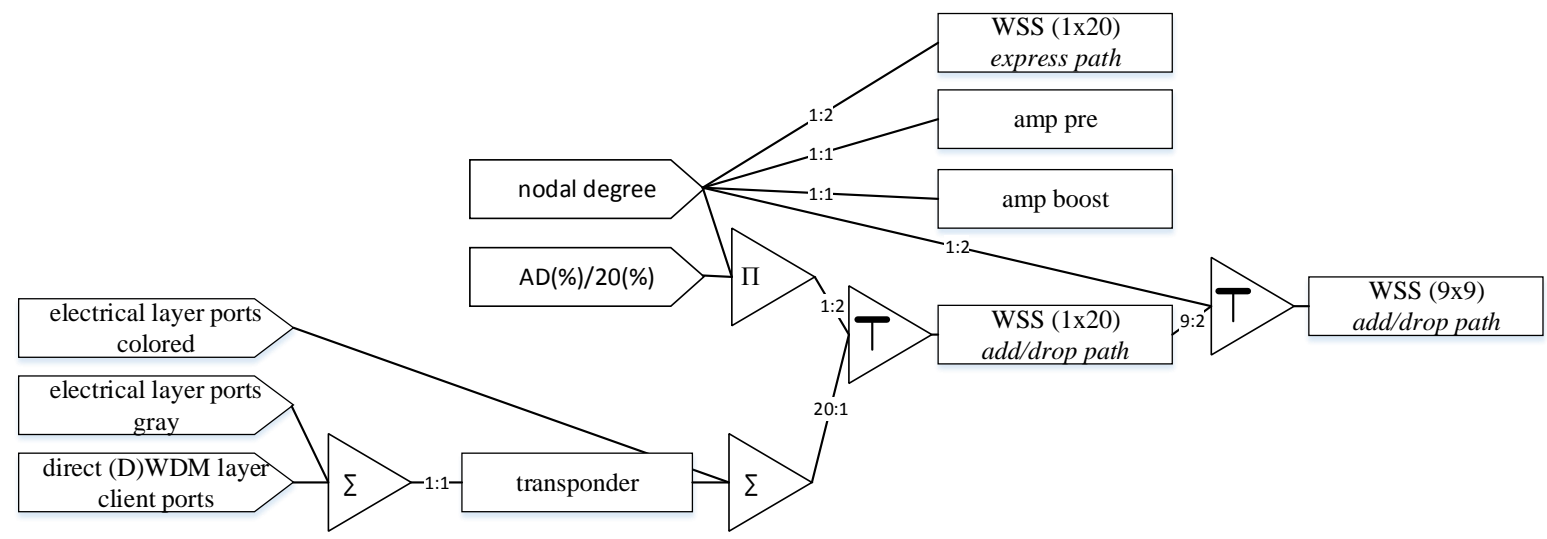

Fig. 6. ECMN model of an optical cross connect: visual notation

be hundreds of kilometers away. A first component is the actual fiber (we assume that dark fiber can be leased). On its path the signal strength decreases due to fiber attenuation. As such an amplifier needs to be installed every $80 \mathrm{~km}$ on average. These amplifiers restore both the original signal as well as noise. To guarantee a sufficient signal-to-noise ratio, dynamic gain equalization (DGE) functionality must be provided at every sixth or seventh amplification site. This is summarized in an ECMN model and illustrated in Figure 7. An overview of price points is given in Table III.

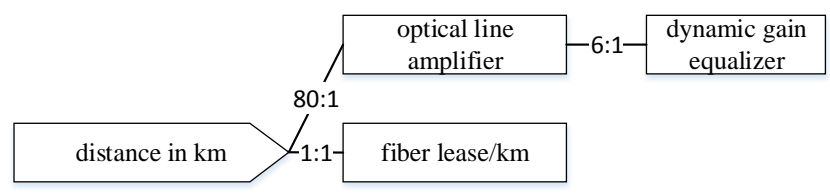

Fig. 7. ECMN model of an optical fiber: visual notation

TABLE III. BUILDING BLOCKS FOR AN OPTICAL FIBER ECMN MODEL

\begin{tabular}{llr}
\hline component & cost & source \\
\hline lease (per km per year) & $2,000.00$ & {$[9]$} \\
optical line amplifire & $7,600.00$ & {$[3]$} \\
dynamic gain equalizer & $8,000.00$ & {$[3]$} \\
\hline
\end{tabular}

We showcase in the next section how to combine the different ECMN models and use them for fast evaluation of network scenarios.

\section{CASE STUdy: Chaining OF IP/MPLS AND WDM ELEMENTS}

This section describes a case study of a carrier network operator who considers expanding its backbone network. To evaluate this network scenario, the ECMN models developed in this paper are used and the advantages of using ECMN models for fast (cost) analysis are highlighted:

- as the output of one model can be the input for a next, ECMN models can be chained to represent complex network scenarios.
- when the network scenario is updated, a mere update of the drivers of the ECMN models suffices to reevaluate the scenario.

\section{A. Scenario Definition}

For this case study, a Belgian backbone network is used (Figure 8). A network operator wants to expand its backbone network and needs an estimate of the extra cost:

- first scenario: the network has 6 multilayer (ML) nodes (these nodes contain building blocs of different layers located in the same physical site, e.g. IP/MPLS in the electrical domain and an OXC based on WSS modules in the optical (DWDM) domain). The nodes are interconnected via leased dark fiber.

- $\quad$ second scenario: the network expands and now has 5 additional multilayer nodes (11 in total). The nodes are interconnected via leased dark fiber.

Services to customers are assumed to be offered by the ML nodes. To estimate the traffic matrices, statistical population data is used from [10]. A peak bandwidth per user of $1 \mathrm{Mbps}$ is assumed and a market share of $10 \%$. As illustrated in 8, Belgium has been subdivided in 11 geographic regions. In the first scenario, the network covers 6 of these regions and the traffic originating from one region is directed towards the others based on the respective share of the population in relation to the total population of the covered regions. Traffic is routed via the least number of hops (when an equal number of hops connects two locations, each path receives an equal share). In the second scenario, all 11 regions are covered and the traffic matrix is established in the same way.

\section{B. ECMN Representation Of A Network}

The IP traffic is routed around the core network assuming an initial IP topology via IP/MPLS routers (Figure 8). Each IP/MPLS node uses 10GbE-interfaces to connect to accessand aggregation equipment. In both scenarios, the network consists out of an integration of IP/MPLS and WDM elements. To interconnect the IP/MPLS router with the OXC, colored interfaces are used, the traffic is multiplexed and is sent to 


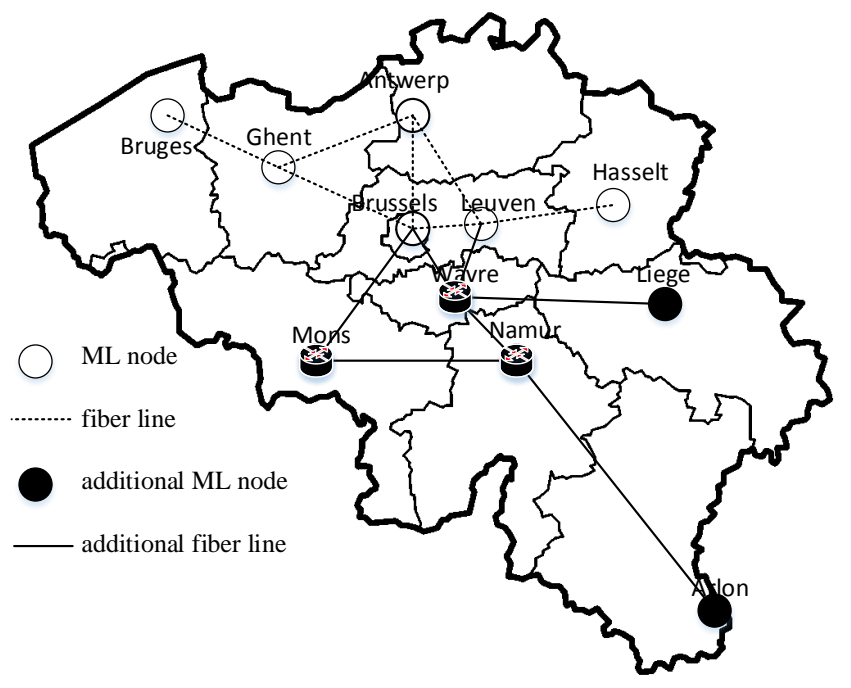

Fig. 8. Topology of the network scenarios under investigation. In the second scenario, the operator expands its transport network southwards.

the add/drop path of the OXC via 100GbE-interfaces. These interfaces are long-haul interfaces which connect the higherlayer nodes directly to the WDM layer (an alternative is to use gray interfaces which require a transponder). The traffic between OXCs is transmitted over optical fiber.

Figure 9 illustrates how the previously discussed ECMN models can be chained together. Of course, initial inputs such as the network topology and traffic matrix are required. Based on these a first set of drivers can be derived (i.e. the nodal degree, the distance between two OXCs and the incoming IP traffic). These serve as input to the drivers which allows to determine the cost of the first ECMN models (i.e. IP/MPLS router). Next, the number of transceivers that need to be installed at the IP/MPLS router are used (i.e. the $100 \mathrm{GbE}$ interfaces) as input driver to the second ECMN model (i.e. OXC) in combination with the nodal degree. Finally the distance between OXCs is used as input to the third ECMN model (i.e. optical fiber). This results in an overview of the number of components as well as the costs at each level (i.e. per ECMN model, per multilayer node, per optical link and of the network as a whole).

To switch between the first and the second scenario, the only change required are an update of the topology and the traffic matrix.

\section{Numerical Results}

Although not the focus of this paper we present the numerical results as summarized in Table IV.

As more ECMN models become available, it will be possible to evaluate other network scenarios (e.g. which involve ECMN models for other types of multilayer nodes, access equipment, caching capacity, etc.). 1 AND 2

\begin{tabular}{|c|c|c|c|}
\hline & cost & scenario 1 & scenario 2 \\
\hline $2 \times 10 \mathrm{GbE}$ line card & $\$ 15,000.00$ & 1 & 4 \\
\hline $4 \times 10 \mathrm{GbE}$ line card & $\$ 35,000.00$ & 1 & 2 \\
\hline $8 \times 10 \mathrm{GbE}$ line card & $\$ 65,000.00$ & 1 & 4 \\
\hline $16 \times 10 \mathrm{GbE}$ line card & $\$ 170,000.00$ & 1 & 1 \\
\hline $24 \times 10 \mathrm{GbE}$ line card & $\$ 400,000.00$ & 2 & 5 \\
\hline $36 \times 10 \mathrm{GbE}$ line card & $\$ 480,000.00$ & 2 & 1 \\
\hline $1 \times 100 \mathrm{GbE}$ line card & $\$ 200,000.00$ & 4 & 11 \\
\hline $2 \times 100 \mathrm{GbE}$ line card & $\$ 400,000.00$ & 7 & 7 \\
\hline basic node 4 slots & $\$ 120,000.00$ & 6 & 11 \\
\hline total cost IP/MPLS & & $\$ 6,365,000.00$ & $\$ 9,360,000.00$ \\
\hline WSS (1x20) express path & $\$ 24,000.00$ & 30 & 58 \\
\hline amp pre & $\$ 3,200.00$ & 15 & 29 \\
\hline amp boost & $\$ 3,200.00$ & 15 & 29 \\
\hline WSS $(1 \times 20)$ add/drop path & $\$ 24,000.00$ & 30 & 58 \\
\hline WSS (9x9) add drop path & $\$ 192,000.00$ & 30 & 58 \\
\hline total cost $O X C$ & & $\$ 7,296,000.00$ & $\$ 14,105,600.00$ \\
\hline lease (per $\mathrm{km}$ per year) & $\$ 2,000.00$ & 6 & 15 \\
\hline dynamic gain equalizer & $\$ 8,000.00$ & 313.4 & 694.9 \\
\hline total cost optical fiber & & $\$ 2,519,200.00$ & $\$ 5,589,200.00$ \\
\hline total costtotal cost & & $\$ 16,180,200.00$ & $\$ 29,054,800.00$ \\
\hline
\end{tabular}

\section{SUMMARY AND FUTURE WORK}

We have introduced a set of requirements and an approach to fast-track techno-economic evaluation of network scenarios such as network planning and network dimensioning. The approach is based on a combination of an easy-to-read visual notation, a set of attributes coupled to the blocks of the visual notation and one or more drivers. Together these form an ECMN model. We presented three of these ECMN models: an IP/MPLS node, an optical link and an OXC. In two examples, these models are chained together and form a multilayer transport network. We show how to obtain the number of components and the costs at different levels of detail and how to transform the first example into the second example without any changes to the ECMN models except from the inputs to the drivers.

In future work, we wish to expand the number of available ECMN models to allow for more diverse network scenarios and to extend the existing tool set to further automate the evaluation (e.g. automatic selection of the most cost-efficient solution from a set of (vendor) alternatives, automatic generation of drivers from a network topology and traffic matrix).

\section{REFERENCES}

[1] K. Casier, M. Van der Wee, S. Vrebrugge, H. Ranaivoson, T. Coenen, and C. Reynders, "Multi-level business modeling and simulation," in $4 e$ International Symposium on Business Modeling and Software Design (BMSD 2014), 2014, pp. 172-179.

[2] K. Casier, M. Van der Wee, and S. Verbrugge, "Cost evaluation of innovative offers using detailed equipment, process and network modeling languages," in Transparent Optical Networks (ICTON), 2014 16th International Conference on. IEEE, 2014, pp. 1-4.

[3] F. Rambach, B. Konrad, L. Dembeck, U. Gebhard, M. Gunkel, M. Quagliotti, L. Serra, and V. López, "A multilayer cost model for metro/core networks," Journal of Optical Communications and Networking, vol. 5, no. 3, pp. 210-225, 2013.

[4] Cisco ASR 9001 Router, Cisco. [Online]. Available: http://www.cisco. com/c/en/us/support/routers/asr-9001-router/model.html

[5] Cisco ASR 9006 Router, Cisco. [Online]. Available: http://www.cisco. com/c/en/us/support/routers/asr-9006-router/model.html

[6] Cisco ASR 9010 Router, Cisco. [Online]. Available: http://www.cisco. $\mathrm{com} / \mathrm{c} / \mathrm{en} / \mathrm{us} / \mathrm{support} /$ routers/asr-9010-router/model.html

[7] Cisco US September 2014 price list, Cisco. 


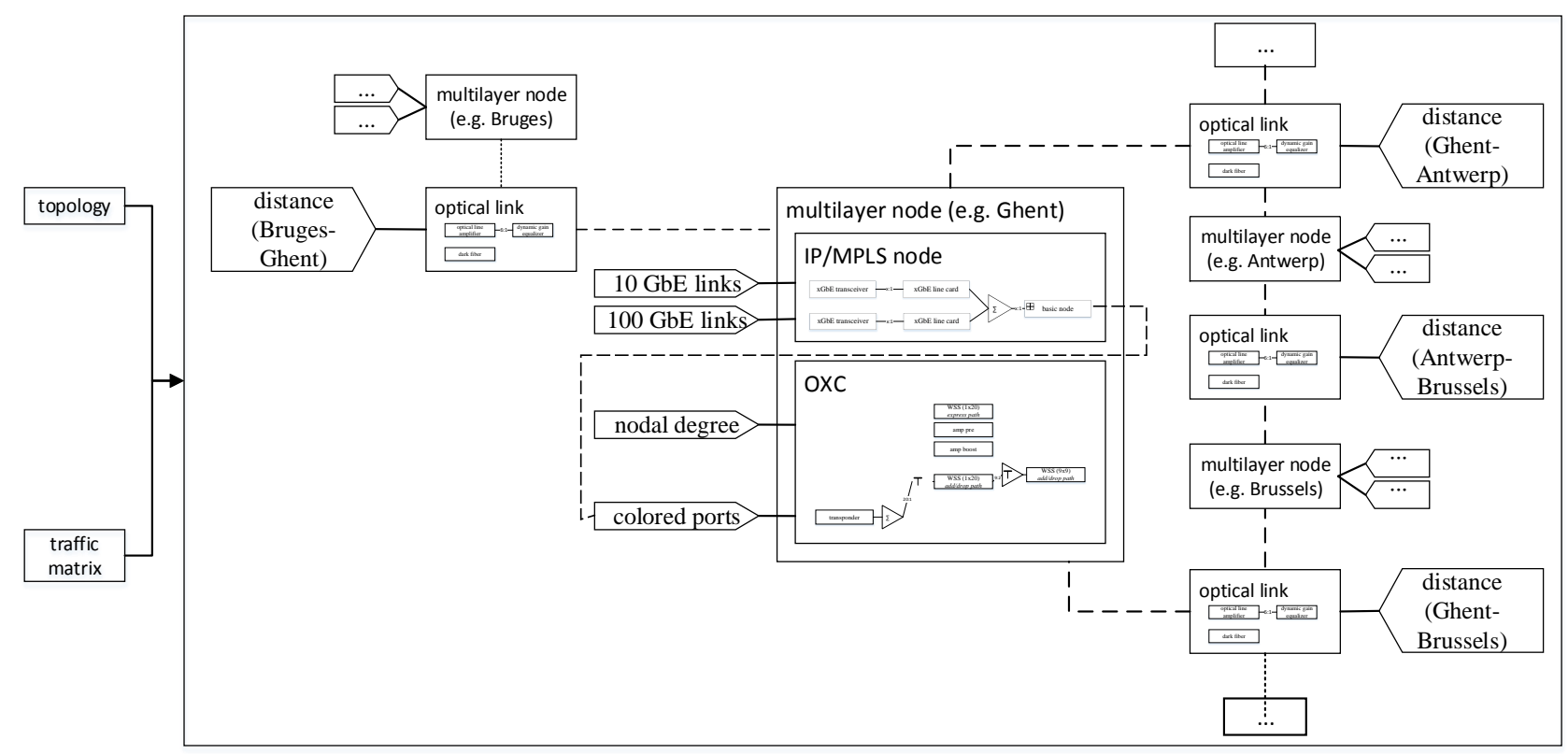

Fig. 9. Overview of the approach to evaluate the different network scenarios via the use of ECMN models. ECMN models can be chained together. In this example an IP/MPLS ECMN model is chained to an OXC ECMN model and two OXC ECMN models are chained via an optical link ECMN model. The drivers are calculated from the given inputs (topology and traffic matrix) and can be updated when an input changes (e.g. between both scenarios).

[8] S. Gringeri, B. Basch, V. Shukla, R. Egorov, and T. J. Xia, "Flexible architectures for optical transport nodes and networks," Communications Magazine, IEEE, vol. 48, no. 7, pp. 40-50, 2010.

[9] K. Christodoulopoulos, M. Angelou, D. Klonidis, P. Zakynthinos, E. Varvarigos, and I. Tomkos, "Value analysis methodology for flexible optical networks," in Proc. of ECOC, 2011.

[10] Bevolkingscijfers per provincie en per gemeente op 1 januari 2015. [Online]. Available: http://www.ibz.rrn.fgov.be/fileadmin/user_upload/ fr/pop/statistiques/population-bevolking-20150101.pdf 